\title{
Comparison of drugs facilitating endoscopy for patients with acute variceal bleeding: a systematic review and network meta- analysis
}

\author{
Ziyuan Zou ${ }^{1,2 \#}$, Xinwen Yan ${ }^{1,2 \#}$, Huanpeng $\mathrm{Lu}^{2 \#}$, Xingshun $\mathrm{Qi}^{3}$, Ye $\mathrm{Gu}^{4}, \mathrm{Xun} \mathrm{Li}^{1}, \mathrm{Bin}^{\mathrm{Wu}^{5}}$, Xiaolong $\mathrm{Qi}^{1}$ \\ ${ }^{1}$ CHESS Center, Institute of Portal Hypertension, The First Hospital of Lanzhou University, Lanzhou 730000, China; ${ }^{2}$ The First School of \\ Clinical Medicine, Nanfang Hospital, Southern Medical University, Guangzhou 510515, China; ${ }^{3}$ Liver Cirrhosis Study Group, Department of \\ Gastroenterology, General Hospital of Shenyang Military Area, Shenyang 110840, China; ${ }^{4}$ Department of Gastroenterology, The Sixth Peoples \\ Hospital of Shenyang, Shenyang 110003, China; ${ }^{5}$ Department of Gastroenterology, The Third Affiliated Hospital of Sun Yat-Sen University, \\ Guangzhou 510630, China \\ Contributions: (I) Conception and design: Z Zou, X Yan, X Qi; (II) Administrative support: X Qi, X Qi, X Li, Y Gu, B Wu; (III) Provision of study \\ materials or patients: Z Zou, X Yan, H Lu; (IV) Collection and assembly of data: Z Zou, X Yan, H Lu; (V) Data analysis and interpretation: Z Zou, X \\ Yan, H Lu, Y Gu, B Wu; (VI) Manuscript writing: All authors; (VII) Final approval of manuscript: All authors. \\ \#These authors contributed equally to this work. \\ Correspondence to: Xiaolong Qi, MD. Professor of Medicine, Chair of CHESS (Chinese Portal Hypertension Diagnosis and Monitoring Study Group), \\ CHESS Center, Institute of Portal Hypertension, The First Hospital of Lanzhou University, Lanzhou 730000, China. Email: qixiaolong@vip.163. \\ com; Bin Wu, MD. Professor of Medicine, Department of Gastroenterology, The Third Affiliated Hospital of Sun Yat-Sen University, Guangzhou \\ 510630, China. Email: wubin6@mail.sysu.edu.cn.
}

Background: We aimed to compare the efficacy of different drugs facilitating endoscopy in patients with acute variceal bleeding.

Methods: Databases were searched to identify randomized controlled trials which compared the efficacy of vasoactive drugs (vasopressin, terlipressin, octreotide, somatostatin) with placebo or each other. The primary outcomes were 6-week and 5-day mortality. Secondary outcomes were 5-day rebleeding, control of initial bleeding and adverse events. Pairwise and network meta-analysis were performed.

Results: We identified 14 RCTs involved 2,187 patients. Four drugs had comparable clinical efficacy in all involving outcomes, except for adverse events. However, we do exhibit a superiority when vasopressin (OR, 4.40; 95\% CI: 1.04-19.57), terlipressin (OR, 4.58; 95\% CI: 1.63-13.63), octreotide (OR, 5.79; 95\% CI: 2.41-16.71) and somatostatin (OR, 5.15; 95\% CI: 1.40-27.39) were compared to placebo respectively as for initial hemostasis. In addition, only octreotide was more effective than placebo in decreasing 5-day rebleeding (OR, 0.44; 95\% CI: 0.22-0.90). Meanwhile, octreotide was shown to have the highest probability ranking the best to improve initial hemostasis (mean rank $=1.8$ ) and carries a lowest risk of adverse events $(9.1 \%)$ and serious adverse events $(0.0 \%)$ compared to other drugs.

Conclusions: Balanced with curative effect and tolerability, octreotide may be the preferred vasoactive drug facilitating endoscopy.

Keywords: Cirrhosis; portal hypertension; vasoconstrictor agents; hemorrhage; endoscopy

Submitted Sep 15, 2019. Accepted for publication Nov 15, 2019.

doi: 10.21037/atm.2019.12.26

View this article at: http://dx.doi.org/10.21037/atm.2019.12.26 


\section{Introduction}

Acute variceal hemorrhage is one of the crucial complications of cirrhosis (1-4). The overall prognosis has been improved over the past few decades on account of significant improvement in the way of diagnosis and therapy, for instance, endoscopic treatment, vasoactive agents and antibiotics $(1,5)$. However, the mortality rate is still growing, which is about $20 \%$ at 6 weeks $(1,3-5)$.

Endoscopic therapy and pharmacological therapy have different mechanisms of action: endoscopic therapy [endoscopic injection sclerotherapy (EIS), endoscopic variceal ligation (EVL)] plays a role directly while vasoactive drugs reduce the portal pressure $(6,7)$. In current practice guidelines, the combined therapeutic procedure of drugs and endoscopy has been highlighted to be the firstline therapy for patients with acute variceal hemorrhage, notably the early application of vasoactive drugs even prior to endoscopic procedure in suspected upper-intestinal hemorrhage (8-10). In the meantime, compared with endoscopic monotherapy, one previous test had proved that, combined therapy in controlling early hemorrhage [relative risk (RR), 1.12; 95\% CI: 1.02-1.23] and 5-day hemostasis (RR, 1.28; 95\% CI: 1.18-1.39) is more effective when vasoactive drug (vasopressin, terlipressin, octreotide or somatostatin) was administrated during the therapeutic course of endoscopic procedure (11).

Nevertheless, a three-arm trial exhibited that somatostatin, terlipressin and octreotide have similar ratio in mortality $(8.0 \%, 8.9 \%$ and $8.8 \%, \mathrm{P}=0.929)$ and hemorrhage $(3.4 \%, 4.8 \%$ and $4.4 \%, \mathrm{P}=0.739)$, which made it difficult to screen out the preferred vasoactive drugs with the highest efficacy to treat acute variceal bleeding (12). Thus, acquiring comparative evidence on pharmacological agents would be very useful. We therefore performed a network meta-analysis integrating direct and indirect evidence to compare the effectiveness of vasoactive drugs (vasopressin, terlipressin, somatostatin, octreotide) facilitating endoscopic therapy for the treatment of acute variceal bleeding in patients with cirrhosis.

\section{Methods}

\section{Study design}

This is a systematic review with pairwise meta-analysis and Bayesian network meta-analysis. The study was carried out according to the Cochrane handbook for systematic reviews of interventions, and reported according to Preferred
Reporting Items for Systematic Reviews and Meta-Analyses (PRISMA) (13). The register on PROSPERO international prospective register of systematic reviews was acquired (CRD42019121039).

\section{Search strategy and study selection}

A comprehensive electronic computerized literature was performed to identify original publications with relevant topic from Web of Science, Medline, Embase, Cochrane Library and Scopus regarding the vasoactive drugs for the treatment of the acute variceal bleeding. We used different terms and various combinations in our search details as reported in Table S1. We searched the literature published before March 2019. All searches were restricted to human studies and only full-text available research was included. Reference lists were manually checked to avoid duplicate.

\section{Eligible criteria}

Eligible research must meet the following criteria:

(I) Study design-RCT;

(II) Study population- Cirrhotic patients with acute gastroesophageal variceal bleeding;

(III) Intervention-Combination of pharmacological therapy (vasopressin or terlipressin or somatostatin or octreotide) and therapeutic endoscopy (EVL or EIS);

(IV) Comparison-Placebo and any active intervention of vasopressin, terlipressin, somatostatin and octreotide;

(V) Endpoints-Mortality or rebleeding.

We excluded:

(I) Non-randomized and observational studies;

(II) Studies, in which the vasoactive drugs were not widely used to treat acute variceal hemorrhage (e.g., vapreotide);

(III) Patients with non-cirrhotic portal hypertension;

(IV) Most of patients known allergy or intolerance to the drugs of the study when included;

(V) Endoscopy-proven bleeding from other sources;

(VI) Without the use of placebo.

\section{Data extraction and risk-of-bias assessment}

Data on the following study-, patient-, and treatmentrelated characteristics were abstracted onto a standardized form: 
(I) Study characteristics: primary author, time period of study/year of publication, patient selection criteria, relevant reported outcomes, and duration of follow-up;

(II) Patient characteristics: age, eligible population, Child-Pugh score, etiology of cirrhosis;

(III) Intervention characteristics: dose and application schedule and the number of patients involved in the trial.

By two authors (Y Xan and Z Zou) independently and discordant results were resolved by discussion between the two authors or by consulting a third senior researcher (X Qi) and resolved by consensus.

We assessed study quality using the Cochrane Collaboration's tool for assessing risk of bias in RCTs (14). Any disagreements were resolved by seeking for consensuses.

\section{Outcomes and definitions}

The following endpoints were included in our network meta-analysis:

(I) Six-week mortality;

(II) 5-day mortality;

(III) 5-day rebleeding;

(IV) Control of initial bleeding within $24 \mathrm{~h}$ after T0;

(V) adverse events.

Time zero (T0) was defined as the time of admission to the first hospital after onset of bleeding or, as the time when bleeding occurred, if the patients was already hospitalized (15). T0 was defined as the time when the patient noticed the clinical sign of upper-intestinal bleeding (haematemesis, melaena or haematochezia of coffee-ground vomitus etc.).

Rebleeding was defined as recurrence of bloody emesis or bright red blood in the nasogastric aspirate with a drop in the $\mathrm{Hb}$ level of more than $1 \mathrm{~g} / \mathrm{dL}$. Control of initial bleeding was defined as:

(I) Absence of hematemesis and melena for 24 consecutive $\mathrm{h}$;

(II) Stable $\mathrm{Hb}$ concentration and hemodynamic conditions for 24 consecutive $\mathrm{h}$ without blood transfusions;

(III) Absence of blood at control endoscopy (15).

\section{Data synthesis and statistical analysis}

Pooled odds ratio (OR) and 95\% confidence intervals (CIs) under fixed model incorporating between-study heterogeneity were used to perform this network metaanalysis (16). We assessed statistical heterogeneity according to $\mathrm{I}^{2}$-index statistic. Cut-offs of $30 \%, 60 \%, 75 \%$ and $>75 \%$ were considered to suggest low, moderate, substantial and considerable heterogeneity respectively (17), and evaluated for publication bias by examining funnel plot asymmetry (18). Direct comparisons were performed using RevMan software (v5.3; Cochrane Collaboration. Copenhagen, Denmark).

In the absence of direct (i.e., head-to-head) comparisons, network meta-analysis was performed within Bayesian framework (19). We modeled any two-arm comparison as a function of each intervention with a reference intervention (i.e., placebo). The hypothesis of consistency or intervention effects underlying this approach which is to say the same effects of direct and indirect comparison are reasonable. The Bayesian network meta-analyses results were compared with pairwise meta-analyses results to evaluate inconsistency. The node splitting method was used to calculate the inconsistency of the model (20). Significant inconsistency was indicated if node-splitting analysis derived $\mathrm{P}<0.05$.

Meanwhile, we assessed the ranking probabilities for all treatments. The treatment hierarchy was summarized and reported as surface under the cumulative ranking curve (SUCRA) values which represent the probabilities of each treatment being ranked the best (21). All $\mathrm{P}$ values are 2-tailed and a $\mathrm{P}<0.05$ indicates a significant difference for all tests (except for heterogeneity).

\section{Statement of ethics approval}

We confirm that all studies involved in data synthesis and statistical analysis in fact get informed consent from each study participants and that each study was approved by an ethics committee or institutional review board.

The authors are accountable for all aspects of the work in ensuring that questions related to the accuracy or integrity of any part of the work are appropriately investigated and resolved.

\section{Results}

One hundred seventy-seven unique studies were identified in total using the search strategy after which we reviewed 60 studies with full-texts and we included 14 RCTs with potential eligibility at last in this network meta-analysis $(12,22-34)$. Literature search process was demonstrated in Figure 1. 

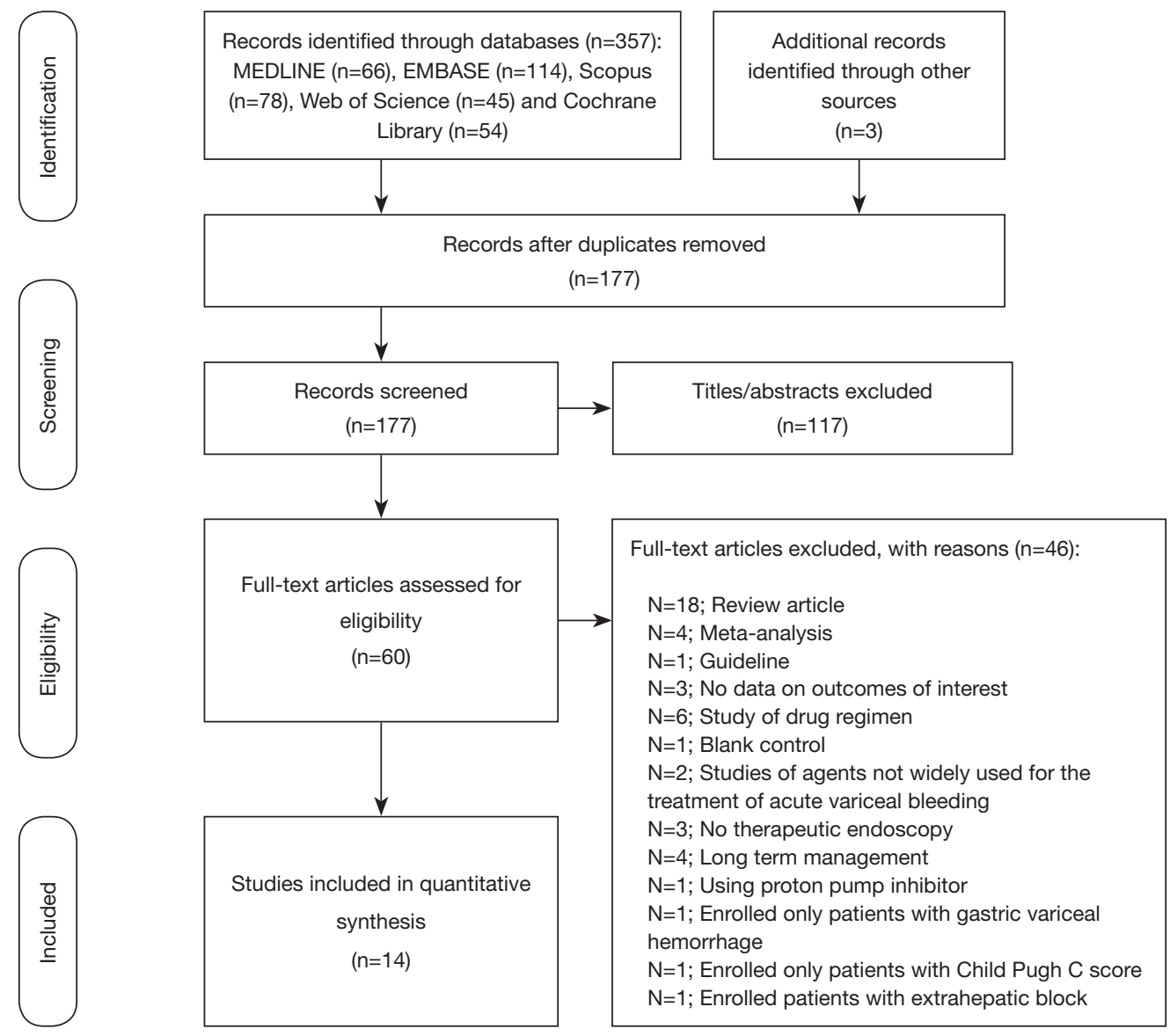

Figure 1 Study selection.

\section{Characteristics of included studies}

Table 1 exhibits the characteristics of the included RCTs in this network meta-analysis. Overall, these 14 trials enrolled 2,203 patients with liver cirrhosis and acute gastroesophageal variceal bleeding, with a median sample size of 65.5 (33-780) patients. Among the RCTs with potential eligibility, the first RCT was published in 1986 and the most recent publication was from 2015. Twelve RCTs $(85.7 \%, 12 / 14)$ were performed as single center trials; the remaining trials were multicenter $(12,24)$. Ten RCTs had regular follow-up time which ranged from 5 to 60 days whereas participants in 4 RCTs were observed until discharged from the hospital.

Of the 14 RCTs comparing the efficacy of pharmacological agents to each other or with placebo on the basis of therapeutic endoscopic intervention, 13 were two-arm trial. Among them:
(I) Octreotide $v s$. placebo, 3 RCTs with 374 patients in total $(24,25,27)$;

(II) Terlipressin $v s$. placebo, 2 RCTs with 110 patients in total $(26,34)$;

(III) Somatostatin vs. placebo, 2 RCTs with 266 patients in total $(22,23)$;

(IV) Octreotide vs. terlipressin, 2 RCTs with 384 patients in total $(31,32)$;

(V) Vasopressin $v s$. terlipressin, 165 patients (28);

(VI) Octreotide $v s$. vasopressin, 48 patients (29);

(VII) Somatostatin vs. octreotide, 33 patients (30);

(VIII) Somatostatin vs. vasopressin, 43 patients (33);

(IX) And the remaining 1 RCT was 3-arm trial with 780 involving patients comparing the efficacy between three pharmacological agents (somatostatin, octreotide, terlipressin) (12). Figure 2 shows the available direct comparison. 


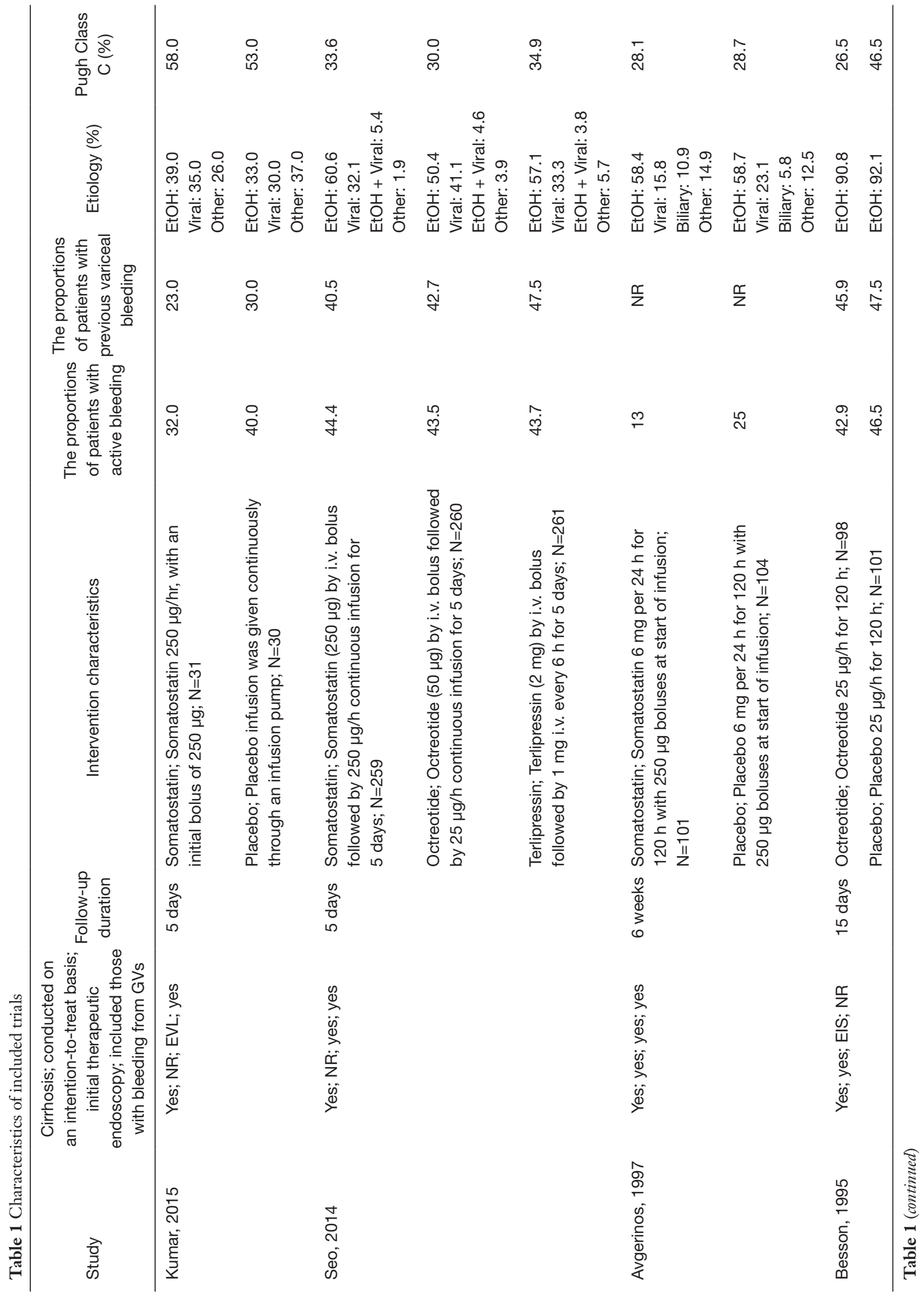




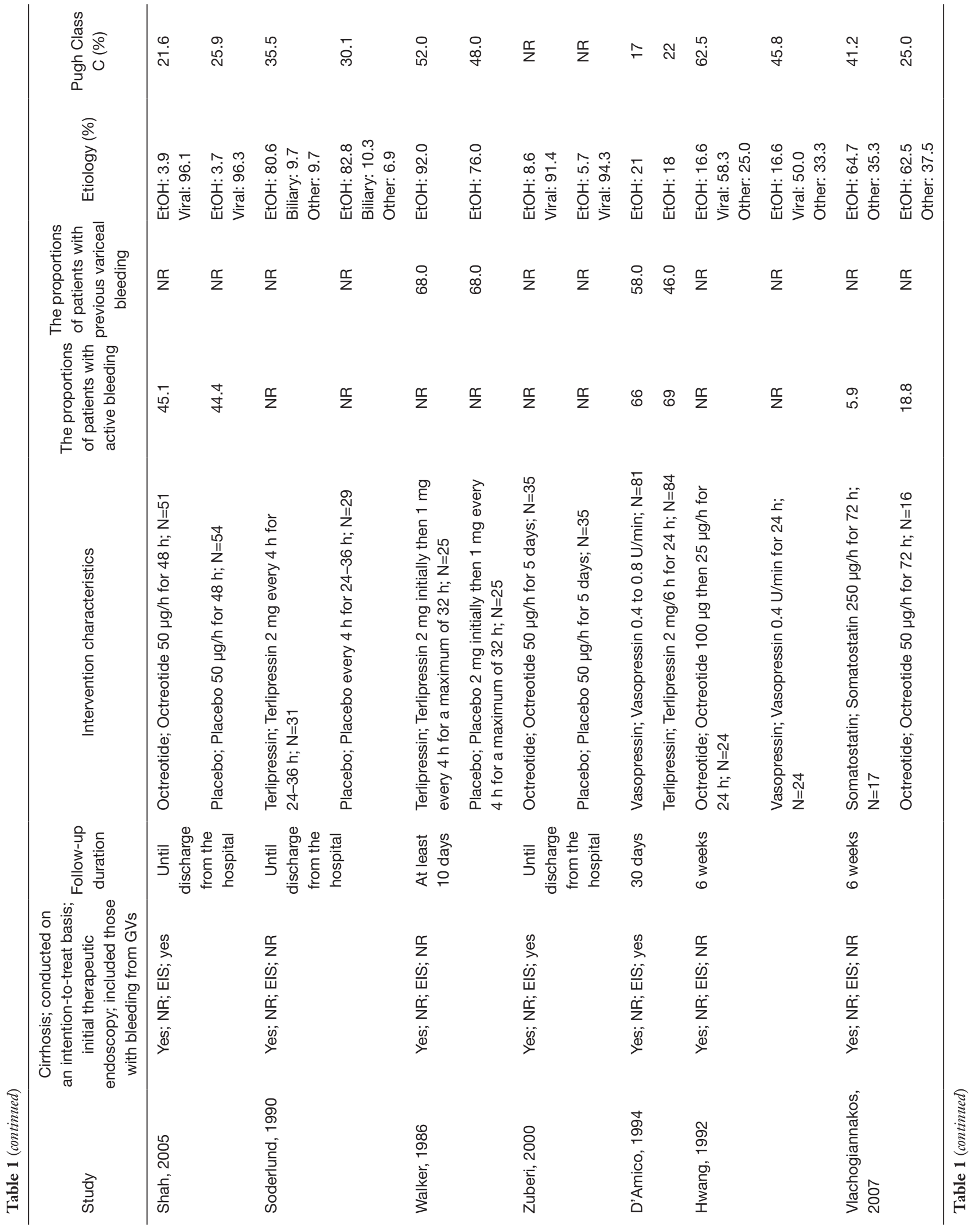




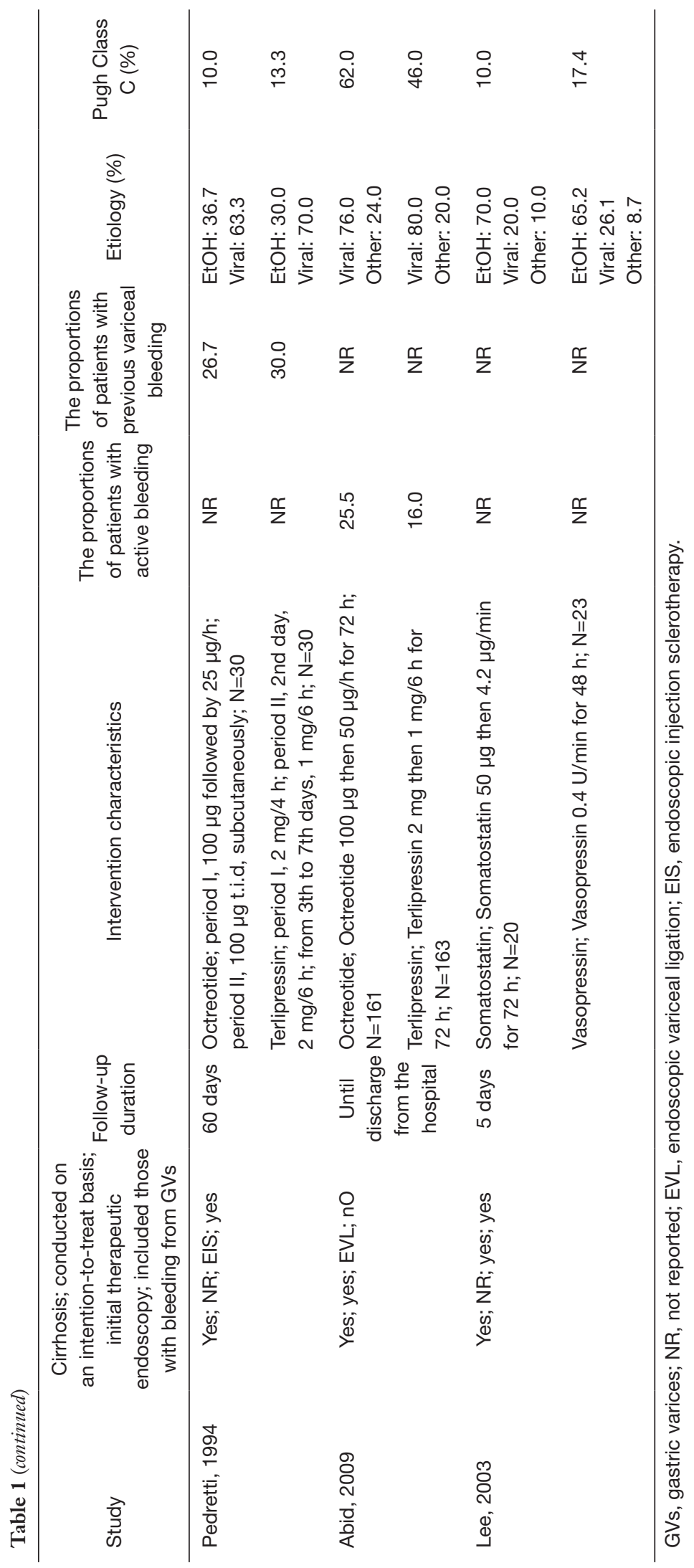



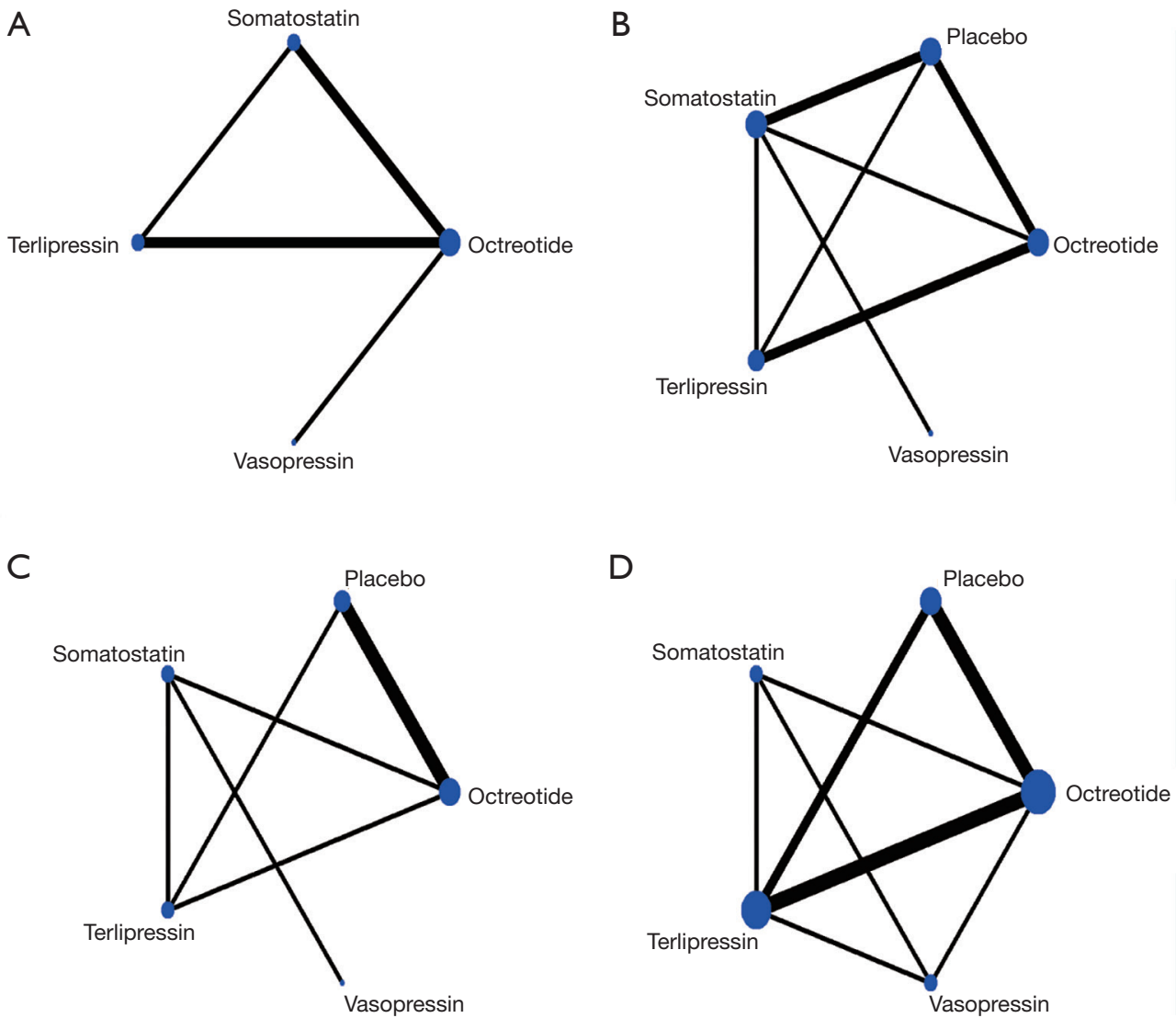

Figure 2 Network geometry of trials for six-week mortality (A), five-day mortality (B), rebleeding (C) and control of initial bleeding (D).

\section{Quality of included studies}

Briefly, the result of quality assessment shows that the studies were felt to be at low risk of bias, with regard to selection, attrition and other bias. Of the 14 included RCTs, 1 RCT $(7.1 \%)$ did not report the method of generation of randomization sequence. Three RCTs (21.4\%) did not provide sufficient details to make a judgement on the adequacy of allocation concealment and another RCT (7.1\%) did not report details on blinding of outcome assessors. Meanwhile, 2 RCTs (14.3\%), 4 RCTs (28.6\%), 5 RCTs $(35.7 \%)$ did not provide sufficient data in the attrition bias, reporting bias and other bias segment respectively. Overall, the risk of bias in individual studies is summarized in Figure S1.

\section{6-week mortality}

\section{Direct meta-analysis}

None of the differences in all comparisons was statistically significant in pairwise meta-analysis as shown in Figure $3 \mathrm{~A}$.

\section{Network meta-analysis}

No agent was clearly superior to others in network metaanalysis (Table 2).

Octreotide had the highest probability ranked the firstbest for improving 6-week mortality (mean rank $=2.1$; SUCRA $=61.9 \%$ ), whereas somatostatin (mean rank $=2.4$; SUCRA $=53.1 \%$ ) and vasopressin (mean rank $=2.4$; SUCRA $=52.7 \%$ ) both had highest probabilities of being ranked second (Table 3).

\section{5-day mortality}

\section{Direct meta-analysis}

None of the differences in all comparisons was statistically significant in pairwise meta-analysis as shown in Figure $3 B$.

Network meta-analysis

None of the difference in all comparisons was statistically 

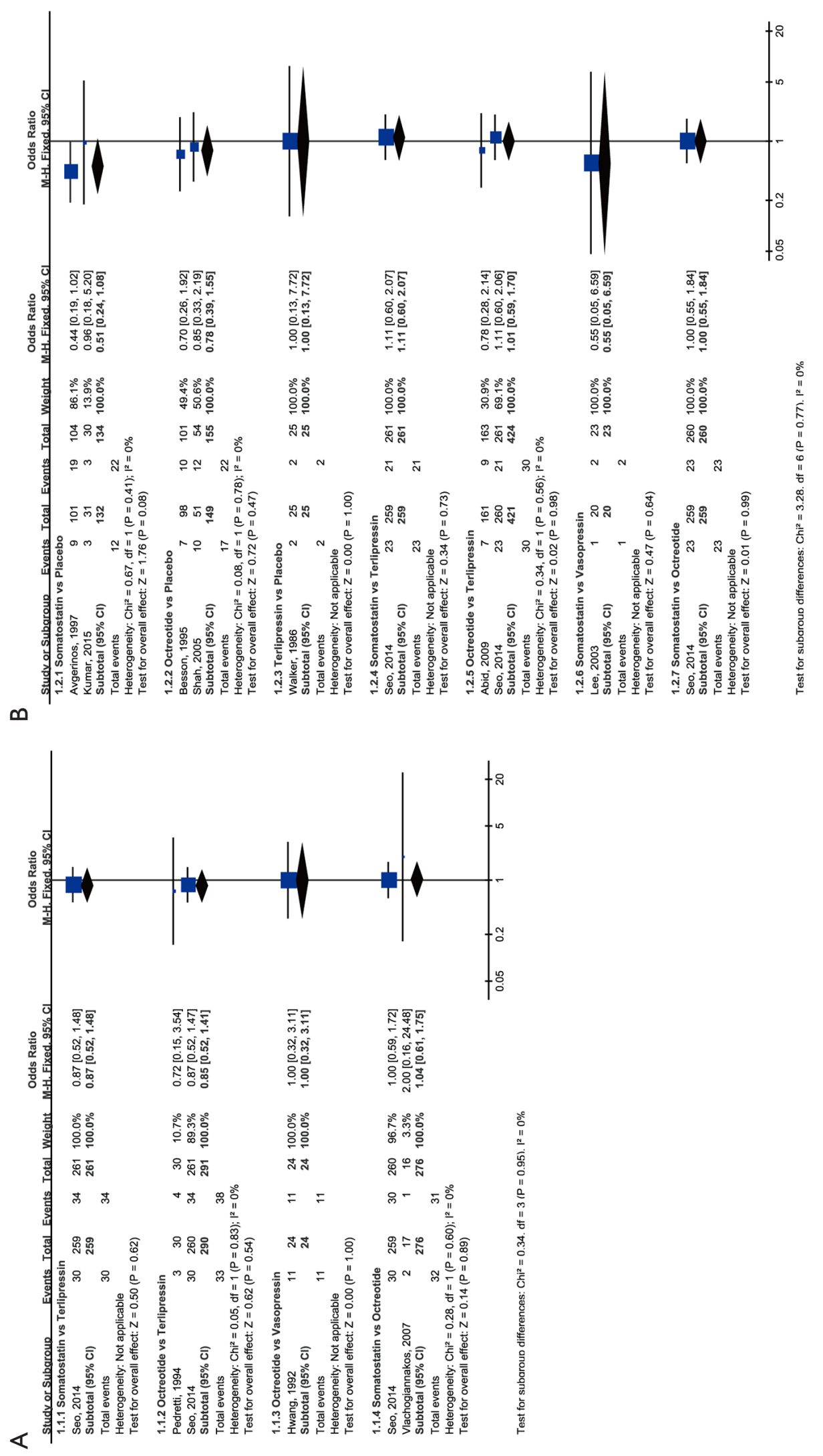


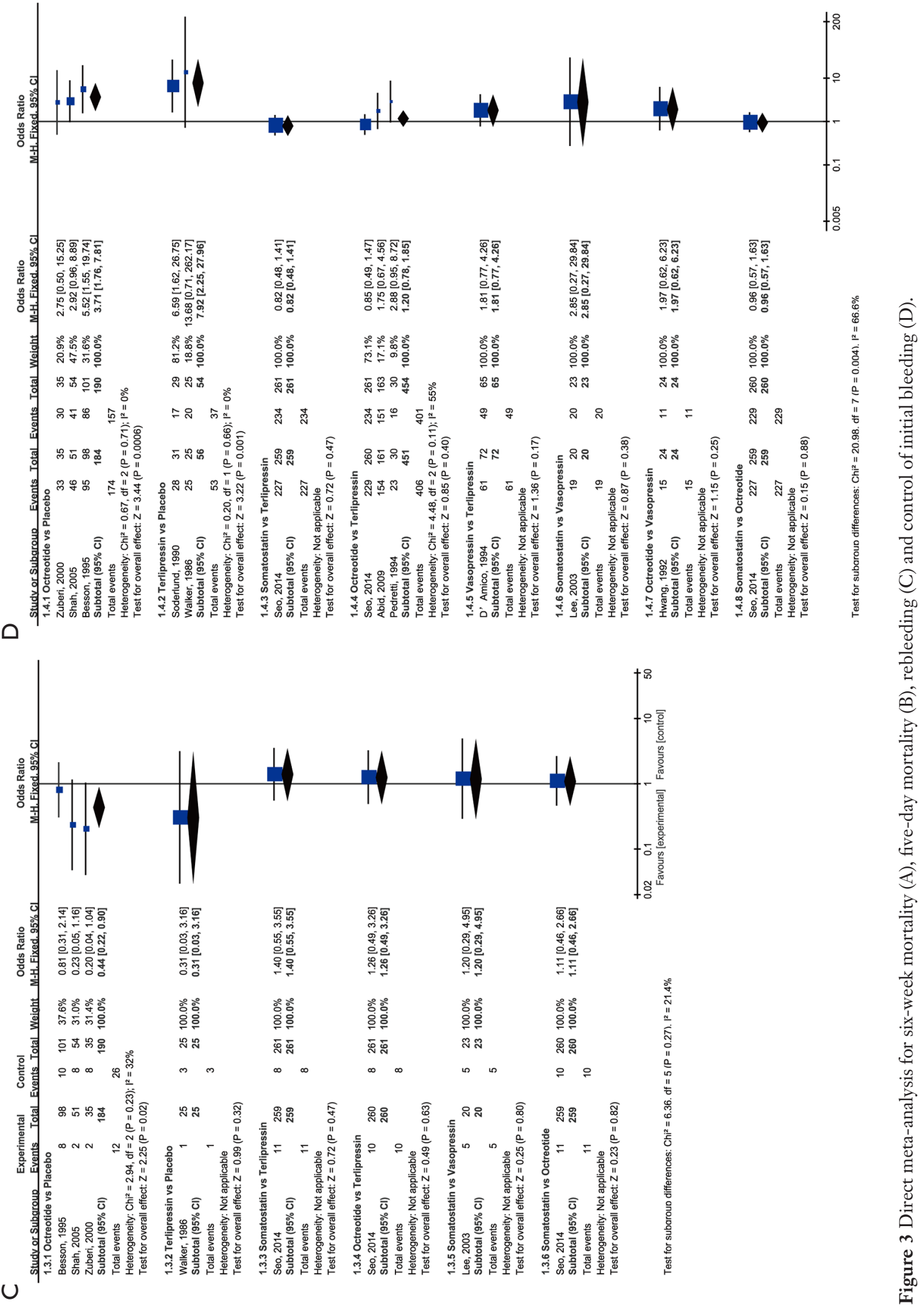


Table 2 Summary of findings reporting the comparative efficacy of pharmacological agents

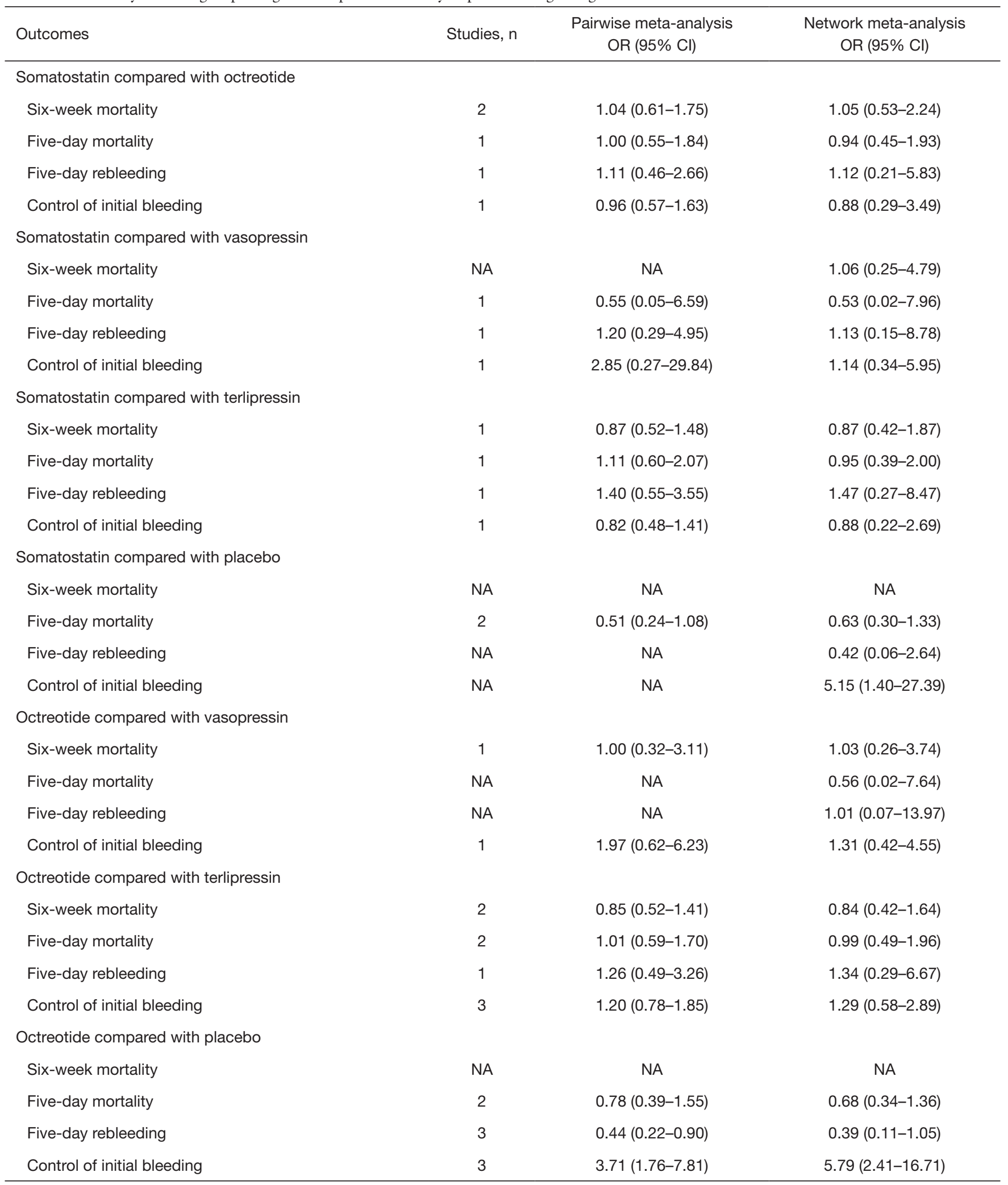

Table 2 (continued) 
Table 2 (continued)

\begin{tabular}{|c|c|c|c|}
\hline Outcomes & Studies, $\mathrm{n}$ & $\begin{array}{c}\text { Pairwise meta-analysis } \\
\text { OR }(95 \% \mathrm{Cl})\end{array}$ & $\begin{array}{c}\text { Network meta-analysis } \\
\text { OR }(95 \% \mathrm{Cl})\end{array}$ \\
\hline \multicolumn{4}{|c|}{ Vasopressin compared with terlipressin } \\
\hline Five-day mortality & NA & NA & $1.81(0.12-43.84)$ \\
\hline \multicolumn{4}{|c|}{ Vasopressin compared with placebo } \\
\hline Six-week mortality & NA & NA & NA \\
\hline Five-day mortality & NA & NA & $1.22(0.08-28.83)$ \\
\hline Six-week mortality & NA & NA & NA \\
\hline Five-day mortality & 1 & $1.00(0.13-7.72)$ & $0.67(0.29-1.70)$ \\
\hline Five-day rebleeding & 1 & $0.31(0.03-3.16)$ & $0.29(0.05-1.38)$ \\
\hline Control of initial bleeding & 2 & $7.92(2.25-27.96)$ & $4.58(1.63-13.63)$ \\
\hline
\end{tabular}

NA, not available.

significant in network meta-analysis (Table 2).

As demonstrated in Table 3, in terms of the efficacy to improve 5-day mortality, somatostatin was shown the most effective (mean rank $=2.2$; SUCRA $=71.0 \%$ ), followed by terlipressin (mean rank $=2.5$; SUCRA $=62.9 \%$ ) and octreotide (mean rank $=2.6$; SUCRA $=60.9 \%$ ).

\section{5-day rebleeding}

\section{Direct meta-analysis}

On direct meta-analysis (Figure 3C), the adjuvant therapy of octreotide facilitating endoscopic intervention for the acute variceal bleeding patients to prevent rebleeding compared to placebo (3 RCTs; OR, 0.44; 95\% CI: 0.22-0.90) was associated with decreased risk of rebleeding. Other comparisons did not illustrate a significant difference.

\section{Network meta-analysis}

None of the difference in all comparisons was statistically significant in network meta-analysis (Table 2).

The ranking of pharmacological agents based on cumulative probability plots and SUCRAs in presented in Table 3. In terms of the efficacy to prevent rebleeding after the initial treatment of acute variceal bleeding within 5 days, terlipressin (mean rank $=2.1$; SUCRA $=71.9 \%$ ), vasopressin ( mean rank $=2.6 ;$ SUCRA $=61.2 \%$ ), octreotide (mean rank $=2.7$; SUCRA $=57.4 \%$ ) respectively had the highest probability ranked the second-best pharmacological agent facilitating therapeutic endoscopy and the least effective was somatostatin (mean rank $=3.0 ;$ SUCRA $=49.4 \%$ ).

\section{Control of initial bleeding within $24 \mathrm{~b}$ after T0}

\section{Direct meta-analysis}

On direct meta-analysis, octreotide (3 RCTs; OR, 3.71; 95\% CI: 1.76-7.81) and terlipressin (2 RCTs; OR, 7.92; 95\% CI: 2.25-27.96) were associated with an increased efficacy for controlling initial bleeding as the adjuvant therapy when compared with placebo. Other comparison did not demonstrate a benefit as shown in Figure 3D.

\section{Network meta-analysis}

In network meta-analysis (Table 2), with the comparison of placebo, octreotide (OR, 5.79; 95\% CI: 2.41-16.71), somatostatin (OR, 5.15; 95\% CI: 1.40-27.39), terlipressin (OR, 4.58; 95\% CI: 1.63-13.63), vasopressin (OR, 4.40; 
Table 3 SUCRA value, probability and mean rank for each treatment

\begin{tabular}{|c|c|c|c|}
\hline Outcomes & $\begin{array}{c}\text { SUCRA value } \\
(\%)\end{array}$ & $\begin{array}{l}\text { Probability best } \\
(\%)\end{array}$ & $\begin{array}{l}\text { Mean } \\
\text { rank }\end{array}$ \\
\hline \multicolumn{4}{|c|}{ Six-week mortality } \\
\hline Somatostatin & 53.1 & 24.8 & 2.4 \\
\hline Octreotide & 61.9 & 24.8 & 2.1 \\
\hline Vasopressin & 52.7 & 42.2 & 2.4 \\
\hline Terlipressin & 32.2 & 8.2 & 3.0 \\
\hline \multicolumn{4}{|c|}{ Five-day mortality } \\
\hline Somatostatin & 71.0 & 30.6 & 2.2 \\
\hline Octreotide & 60.9 & 17.3 & 2.6 \\
\hline Vasopressin & 35.4 & 26.9 & 3.6 \\
\hline Terlipressin & 62.9 & 24.1 & 2.5 \\
\hline Placebo & 19.8 & 1.1 & 4.2 \\
\hline \multicolumn{4}{|c|}{ Five-day rebleeding } \\
\hline Somatostatin & 49.4 & 8.1 & 3.0 \\
\hline Octreotide & 57.4 & 16.2 & 2.7 \\
\hline Vasopressin & 61.2 & 37.4 & 2.6 \\
\hline Terlipressin & 71.9 & 38.2 & 2.1 \\
\hline Placebo & 10.1 & 0.1 & 4.6 \\
\hline \multicolumn{4}{|c|}{ Control of initial bleeding } \\
\hline Somatostatin & 60.7 & 23.8 & 2.6 \\
\hline Octreotide & 80.5 & 45.3 & 1.8 \\
\hline Vasopressin & 58.5 & 23.8 & 2.7 \\
\hline Terlipressin & 49.7 & 7.1 & 3.0 \\
\hline Placebo & 0.6 & 0.0 & 5.0 \\
\hline
\end{tabular}

95\% CI: 1.04-19.57) respectively presented more effective to control the initial bleeding as adjuvant therapy. No other comparison reached statistical significance.

As presented in Table 3, as for the efficacy to control initial bleeding as the adjuvant therapy, octreotide (mean rank $=1.8$; SUCRA $=80.5 \%$ ) was the most effective agent. Somatostatin (mean rank $=2.6$; SUCRA $=60.7 \%$ ) and vasopressin (mean rank $=2.7$; SUCRA $=58.5 \%$ ) had the highest probability of being ranked the second and the least effective was terlipressin (mean rank $=3.0$; SUCRA $=49.7 \%$ ).

\section{Adverse events}

Owing to the fact that the definitions of serious adverse
Table 4 Adverse events

\begin{tabular}{lccc}
\hline Interventions & $\begin{array}{c}\text { Total adverse } \\
\text { events, } \mathrm{n}(\%)\end{array}$ & $\begin{array}{c}\text { Serious adverse } \\
\text { events, } \mathrm{n}(\%)\end{array}$ & $\begin{array}{c}\text { Total } \\
\text { patients, (n) }\end{array}$ \\
\hline Somatostatin & $59(14.4)$ & $2(0.5)$ & 408 \\
Octreotide & $30(9.1)$ & $0(0.0)$ & 330 \\
Vasopressin & $60(57.1)$ & $7(6.7)$ & 105 \\
Terlipressin & $106(24.6)$ & $3(0.7)$ & 431 \\
\hline
\end{tabular}

events were different among the involved researches, thus we were unable to perform an analysis for SAEs. Table 4 shows all the reported adverse events by treatment arm. Octreotide had the least number of both reported adverse events $(9.1 \%)$ and severe adverse events $(0.0 \%)$.

\section{Publication bias and Network Coberence}

We did not find evidence of publication bias based on funnel plot asymmetry (Figure S2). In addition, Nodesplitting analysis indicated that there was no significant inconsistency between direct and indirect evidence in all involving outcome (all $\mathrm{P}>0.05$ ).

\section{Discussion}

Not only can vasoactive drug require no complex technique but avoid the hazards of invasive methods, which make it appealing in controlling acute variceal bleeding. By the method of enzymatic cleavage, terlipressin, the analogue of vasopressin, is slowly converted into active vasopressin in vivo. With less severe vascular complications and their subsequent discontinuation of therapy, terlipressin have shown to be much more effective than vasopressin in controlling variceal bleeding with an extension of the biological activity (35-37).

In the meantime, the mechanism of somatostatin and its analogue octreotide is the visceral vasoconstriction of the collateral vessels, which can ameliorate the collateral blood flow and have the similar pharmacological effects for variceal hemorrhage (38-40). In addition, on the one hand, octreotide could reduce gastroduodenal mucosal flow and upregulate intragastric $\mathrm{pH}$ (41), on the other hand, octreotide is generally well tolerated with transient or mild adverse events (42) mostly occurring in gastrointestinal tract and hepatic duct and related to the inhibition the release of multiple hormones in gastrointestinal tract $(43,44)$.

In this updated systematic review and network meta- 
analysis, we combined direct and indirect evidence from 14 RCTs involving 2,187 patients with gastroesophageal varices to estimate the comparative efficacy of pharmacological agents as adjuvant therapy to facilitate endoscopic interventions. We made several key observations:

(I) Vasopressin, terlipressin, somatostatin and octreotide were all prior to placebo with statistically significant difference with respect to initial control of bleeding;

(II) Octreotide had the highest probability of being ranked the first as for the outcome of initial control of hemorrhage with mean rank equals to 1.8 which is the only statistic of all comparison between the interval;

(III) In the pairwise meta-analysis of 5-day rebleeding, only octreotide was shown to be more effective over placebo.

In our network meta-analysis, when comparing the aforementioned four pharmacological agents, we do not appreciate a statistical difference in 6-week mortality, initial control of bleeding, 5-day mortality and 5-day rebleeding. In the meantime, given that none of the vasoactive drugs were associated with statistically significant difference when compared with placebo in the outcome of 6-week mortality, thus indicating the necessity of other pharmacological agents such as carvedilol or propranolol once vasoactive drugs are discontinued. Nevertheless, we do exhibit a superiority when these drugs were compared to placebo and note that octreotide had the highest probability of being ranked the first as for the initial control of hemostasis with mean rank equals to 1.8. Because of the variable definition of specific side effects in the included studies, we did not analyze these in network meta-analysis. Instead, we calculated the total adverse events to exhibit a general evaluation of the safety and tolerability. As a result, octreotide carried the lowest risk of adverse events and serious adverse events compared to other drugs.

It had been illustrated in previous study that riskstratification model consists of Child-Pugh classification or End-Stage-Liver Disease (MELD) score and clinical manifestation and index including initial systolic blood pressure and serum creatinine are associated with therapeutic effect among those patients who presenting with acute variceal bleeding $(12,45,46)$. Apparently, all these parameters need to be taken into account to tailor the therapeutic strategy for individual treatment on the basis of the comparative results from previous trials. Endoscopic monotherapy and short-term pharmacological therapy can be administrated to patients with low-risk variceal bleeding in avoidance of unnecessary side effects, whereas additional methods of portal pressure reduction such as transjugular intrahepatic portosystemic shunt procedure rendering sufficient and efficient benefit for high-risk patients (47).

Besides inherent limitations of individual trials, there are limitations to our analyses. The doses and administration of the medications differed among the studies, thus possibly producing bias. Meanwhile, the most widely used endoscopic treatment administrated to patients with suspected variceal bleeding were EIS and EVL. Though EVL is technically a superior endoscopic procedure with better results in acute bleeding $(48,49)$, we did not strictly impose restrictions on the choice of endoscopy treatment. Despite of the fact that EIS and vasopressin were no longer used and recommended for acute variceal bleeding, they were hired as spots in Bayesian model in this network metaanalysis. Additionally, there were distinctions of methods of outcome ascertainment among involved trials.

The main assumption underpinning the transitivity of direct evidence and indirect evidence is that there exists the common comparator between the trials (50). In avoidance of the inconsistency, we strictly followed the practice guideline (51) recommendation of the initial endoscopy procedure if the detection of acute variceal bleeding when selecting fulfilling trials. Overall, this systematic review indicated that octreotide provides the best initial hemostasis efficacy, followed by somatostatin and vasopressin, and thirdly by terlipressin. Moreover, only octreotide was more effective than placebo in decreasing 5 -day rebleeding. Considering the benefit needs to be balanced against safety and tolerability, octreotide also carries the lowest risk of adverse events and severe adverse events. Taken together, octreotide is rendered as the preferred vasoactive drugs for patients with the detection of acute variceal bleeding.

\section{Acknowledgments}

Funding: This work was supported by the grants from National Natural Science Foundation of China (81600510); Guangdong Science Fund for Distinguished Young Scholars (2018B030306019); Guangzhou IndustryAcademia-Research Collaborative Innovation Major Project (201704020015).

\section{Footnote}

Conflicts of Interest: The authors have no conflicts of interest to declare. 
Ethical Statement: The authors are accountable for all aspects of the work in ensuring that questions related to the accuracy or integrity of any part of the work are appropriately investigated and resolved.

\section{References}

1. Ibrahim M, Mostafa I, Deviere J. New Developments in Managing Variceal Bleeding. Gastroenterology 2018;154:1964-9.

2. Bosch J, Iwakiri Y. The portal hypertension syndrome: etiology, classification, relevance, and animal models. Hepatol Int 2018;12:1-10.

3. Ibrahim M, El-Mikkawy A, Abdel Hamid M, et al. Early application of haemostatic powder added to standard management for oesophagogastric variceal bleeding: a randomised trial. Gut 2019;68:844-53.

4. Garcia-Pagan J, Patch D. Trials and Tribulations: The Prevention of Variceal Rebleeding. Gastroenterology 2015;149:528-31.

5. Sauerbruch T, Mengel M, Dollinger M, et al. Prevention of Rebleeding From Esophageal Varices in Patients With Cirrhosis Receiving Small-Diameter Stents Versus Hemodynamically Controlled Medical Therapy. Gastroenterology 2015;149:660-8.e1.

6. Lo GH. Endoscopic treatments for portal hypertension. Hepatology International 2018;12:91-101.

7. Baiges A, Hernandez-Gea V, Bosch J. Pharmacologic prevention of variceal bleeding and rebleeding. Hepatology International 2018;12:68-80.

8. Garcia-Tsao G, Bosch J. Management of varices and variceal hemorrhage in cirrhosis. $\mathrm{N}$ Engl $\mathrm{J}$ Med 2010;362:823-32.

9. de Franchis R. Evolving consensus in portal hypertension. Report of the Baveno IV consensus workshop on methodology of diagnosis and therapy in portal hypertension. J Hepatol 2005;43:167-76.

10. Garcia-Tsao G, Sanyal A, Grace N, et al. Prevention and management of gastroesophageal varices and variceal hemorrhage in cirrhosis. Hepatology 2007;46:922-38.

11. Banares R, Albillos A, Rincon D, et al. Endoscopic treatment versus endoscopic plus pharmacologic treatment for acute variceal bleeding: a meta-analysis. Hepatology 2002;35:609-15.

12. Seo YS, Park SY, Kim MY, et al. Lack of difference among terlipressin, somatostatin, and octreotide in the control of acute gastroesophageal variceal hemorrhage. Hepatology 2014;60:954-63.
13. Liberati A, Altman DG, Tetzlaff J, et al. The PRISMA statement for reporting systematic reviews and metaanalyses of studies that evaluate healthcare interventions: explanation and elaboration. BMJ 2009;339:b2700.

14. Higgins JP, Altman DG, Gotzsche PC, et al. The Cochrane Collaboration's tool for assessing risk of bias in randomised trials. BMJ 2011;343:d5928.

15. de Franchis R, Pascal JP, Ancona E, et al. Definitions, methodology and therapeutic strategies in portal hypertension. A Consensus Development Workshop, Baveno, Lake Maggiore, Italy, April 5 and 6, 1990. J Hepatol 1992;15:256-61.

16. DerSimonian R, Laird N. Meta-analysis in clinical trials. Control Clin Trials 1986;7:177-88.

17. Higgins JP, Thompson SG, Deeks JJ, et al. Measuring inconsistency in meta-analyses. BMJ 2003;327:557-60.

18. Egger M, Davey S, Schneider M, et al. Bias in metaanalysis detected by a simple, graphical test. BMJ 1997;315:629-34.

19. Dias S, Welton NJ, Sutton AJ, et al. Evidence synthesis for decision making 4: inconsistency in networks of evidence based on randomized controlled trials. Med Decis Making 2013;33:641-56.

20. Dias S, Welton NJ, Caldwell DM, et al. Checking consistency in mixed treatment comparison meta-analysis. Stat Med 2010;29:932-44.

21. Salanti G, Ades AE, Ioannidis JP. Graphical methods and numerical summaries for presenting results from multipletreatment meta-analysis: an overview and tutorial. J Clin Epidemiol 2011;64:163-71.

22. Kumar A, Jha SK, Mittal VV, et al. Addition of Somatostatin After Successful Endoscopic Variceal Ligation Does not Prevent Early Rebleeding in Comparison to Placebo: A Double Blind Randomized Controlled Trial. J Clin Exp Hepatol 2015;5:204-12.

23. Avgerinos A, Nevens F, Raptis S, et al. Early administration of somatostatin and efficacy of sclerotherapy in acute oesophageal variceal bleeds: the European Acute Bleeding Oesophageal Variceal Episodes (ABOVE) randomised trial. Lancet 1997;350:1495-9.

24. Besson I, Ingrand P, Person B, et al. Sclerotherapy with or without octreotide for acute variceal bleeding. $\mathrm{N} \mathrm{Engl} \mathrm{J}$ Med 1995;333:555-60.

25. Shah HA, Mumtaz K, Jafri W, et al. Sclerotherapy plus octreotide versus sclerotherapy alone in the management of gastro-oesophageal variceal hemorrhage. J Ayub Med Coll Abbottabad 2005;17:10-4.

26. Soderlund C, Magnusson I, Torngren S, et al. Terlipressin 


\section{Page 16 of 17}

(triglycyl-lysine vasopressin) controls acute bleeding oesophageal varices. A double-blind, randomized, placebo-controlled trial. Scand J Gastroenterol 1990;25:622-30.

27. Zuberi BF, Baloch Q. Comparison of endoscopic variceal sclerotherapy alone and in combination with octreotide in controlling acute variceal hemorrhage and early rebleeding in patients with low-risk cirrhosis. Am J Gastroenterol 2000;95:768-71.

28. D'Amico G, Traina M, Vizzini G, et al. Terlipressin or vasopressin plus transdermal nitroglycerin in a treatment strategy for digestive bleeding in cirrhosis. A randomized clinical trial. Liver Study Group of V. Cervello Hospital. J Hepatol 1994;20:206-12.

29. Hwang SJ, Lin HC, Chang CF, et al. A randomized controlled trial comparing octreotide and vasopressin in the control of acute esophageal variceal bleeding. J Hepatol 1992;16:320-5.

30. Vlachogiannakos J, Kougioumtzian A, Triantos C, et al. Clinical trial: The effect of somatostatin vs. octreotide in preventing post-endoscopic increase in hepatic venous pressure gradient in cirrhotics with bleeding varices. Aliment Pharmacol Ther 2007;26:1479-87.

31. Pedretti G, Elia G, Calzetti C, et al. Octreotide versus terlypressin in acute variceal hemorrhage in liver cirrhosis. Emergency control and prevention of early rebleeding. Clin Investig 1994;72:653-9.

32. Abid S, Jafri W, Hamid S, et al. Terlipressin vs. octreotide in bleeding esophageal varices as an adjuvant therapy with endoscopic band ligation: a randomized doubleblind placebo-controlled trial. Am J Gastroenterol 2009;104:617-23.

33. Lee HY, Lee HJ, Lee SM, et al. A prospective randomized controlled clinical trial comparing the effects of somatostatin and vasopressin for control of acute variceal bleeding in the patients with liver cirrhosis. Korean J Intern Med 2003;18:161-6.

34. Walker S, Stiehl A, Raedsch B, et al. Terlipressin in bleeding esophageal varices: a placebo-controlled, doubleblind study. Hepatology 1986;6:112-5.

35. Freeman J, Cobden I, Lishman A, et al. Controlled trial of terlipressin ('Glypressin') versus vasopressin in the early treatment of oesophageal varices. Lancet 1982;2:66-8.

36. Arora V, Maiwall R, Rajan V, et al. Terlipressin Is Superior to Noradrenaline in the Management of Acute Kidney Injury in Acute on Chronic Liver Failure. Hepatology 2018. [Epub ahead of print].
37. Walker S, Kreichgauer HP, Bode JC. Terlipressin vs. somatostatin in bleeding esophageal varices: a controlled, double-blind study. Hepatology 1992;15:1023-30.

38. Huang HC, Lee FY, Chan CC, et al. Effects of somatostatin and octreotide on portal-systemic collaterals in portal hypertensive rats. J Hepatol 2002;36:163-8.

39. Sakurabayashi S, Koh KC, Chen L, et al. Octreotide ameliorates the increase in collateral blood flow during postprandial hyperemia in portal hypertensive rats. J Hepatol 2002;36:507-12.

40. Wiest R, Tsai MH, Groszmann RJ. Octreotide potentiates PKC-dependent vasoconstrictors in portal-hypertensive and control rats. Gastroenterology 2001;120:975-83.

41. Kubba AK, Dallal H, Haydon GH, et al. The effect of octreotide on gastroduodenal blood flow measured by laser Doppler flowmetry in rabbits and man. Am J Gastroenterol 1999;94:1077-82.

42. Bornschein J, Drozdov I, Malfertheiner P. Octreotide LAR: safety and tolerability issues. Expert Opin Drug Saf 2009;8:755-68.

43. Lamberts SW, van der Lely AJ, de Herder WW, et al. Octreotide. N Engl J Med 1996;334:246-54.

44. Chandok N, Kamath PS, Blei A, et al. Randomised clinical trial: the safety and efficacy of long-acting octreotide in patients with portal hypertension. Aliment Pharmacol Ther 2012;35:904-12.

45. Reverter E, Tandon P, Augustin S, et al. A MELDbased model to determine risk of mortality among patients with acute variceal bleeding. gastroenterology 2014;146:412-9.e3.

46. Amitrano L, Guardascione MA, Manguso F, et al. The effectiveness of current acute variceal bleed treatments in unselected cirrhotic patients: refining shortterm prognosis and risk factors. Am J Gastroenterol 2012;107:1872-8.

47. Hernandez-Gea V, Berbel C, Baiges A, et al. Acute variceal bleeding: risk stratification and management (including TIPS). Hepatol Int 2018;12:81-90.

48. Villanueva C, Piqueras M, Aracil C, et al. A randomized controlled trial comparing ligation and sclerotherapy as emergency endoscopic treatment added to somatostatin in acute variceal bleeding. J Hepatol 2006;45:560-7.

49. Lo GH, Lai KH, Cheng JS, et al. Emergency banding ligation versus sclerotherapy for the control of active bleeding from esophageal varices. Hepatology 1997;25:1101-4.

50. Donegan S, Williamson P, D'Alessandro U, et al. Assessing 
key assumptions of network meta-analysis: a review of methods. Res Synth Methods 2013;4:291-323.

51. de Franchis R. Expanding consensus in portal

Cite this article as: Zou Z, Yan X, Lu H, Qi X, Gu Y, Li X, Wu B, Qi X. Comparison of drugs facilitating endoscopy for patients with acute variceal bleeding: a systematic review and network meta-analysis. Ann Transl Med 2019;7(23):717. doi: 10.21037/atm.2019.12.26 hypertension: Report of the Baveno VI Consensus Workshop: Stratifying risk and individualizing care for portal hypertension. J Hepatol 2015;63:743-52. 


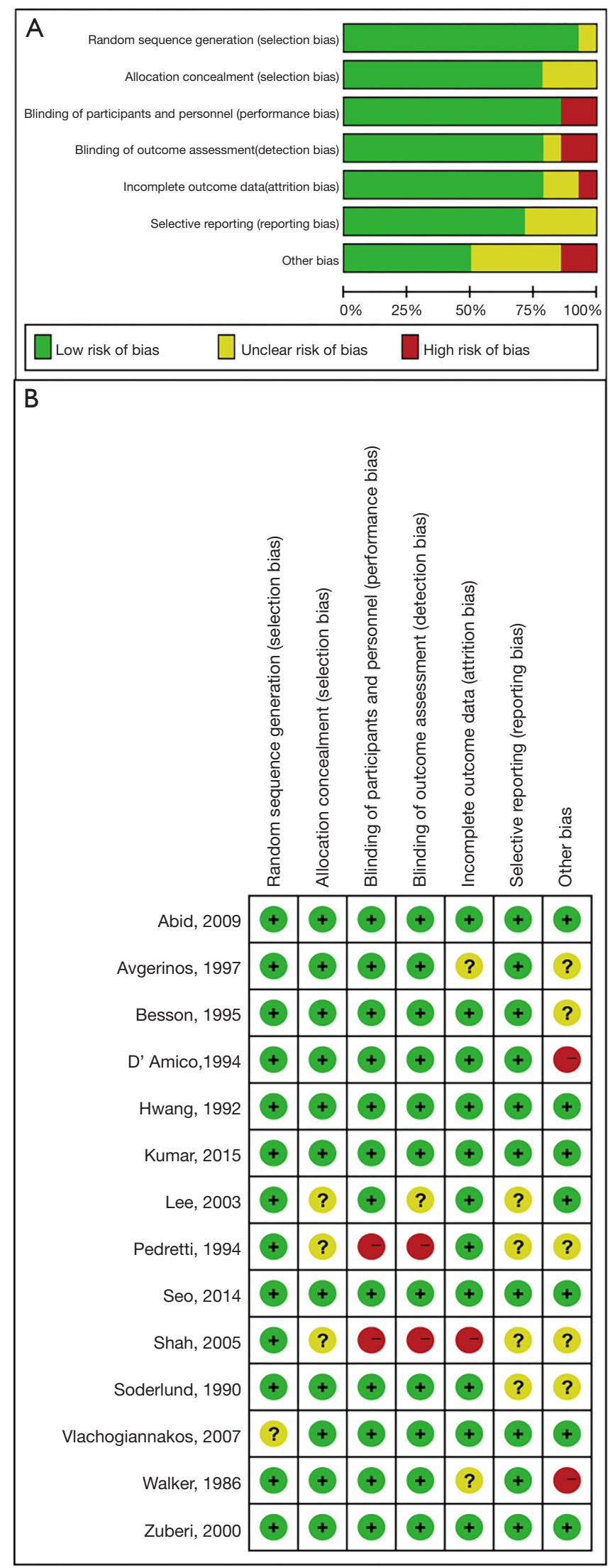

Figure S1 Quality assessment of included studies.
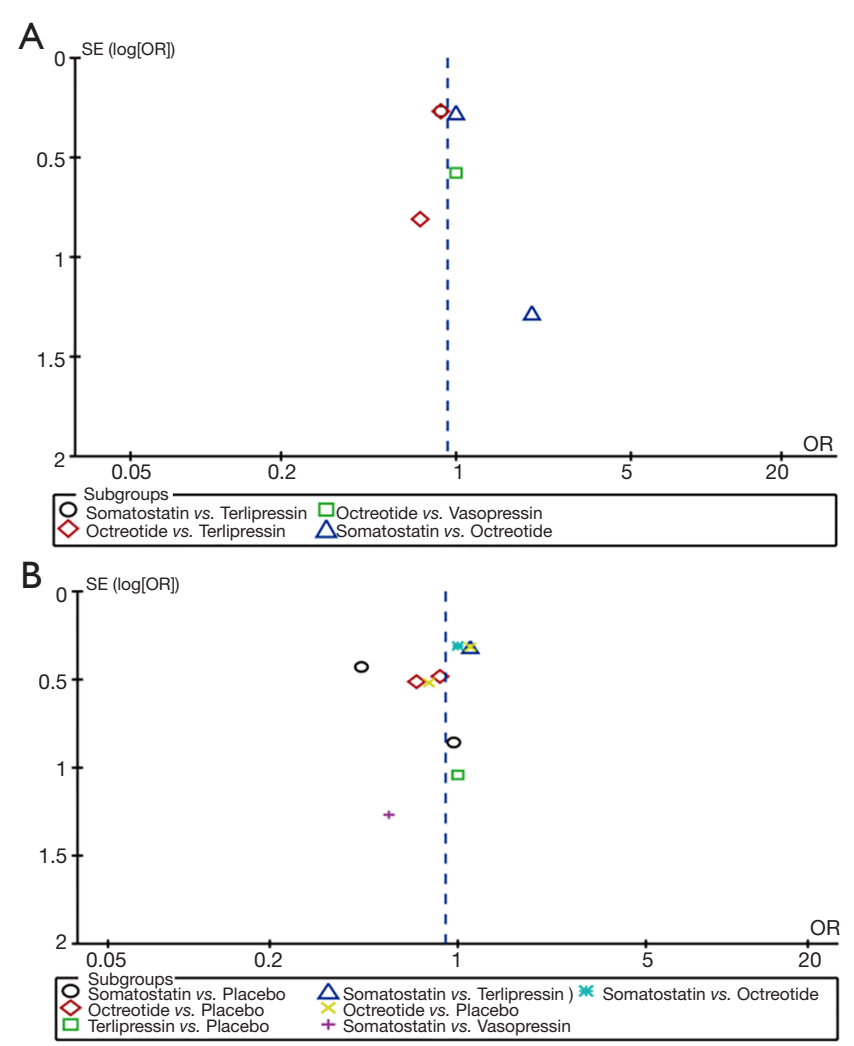

C

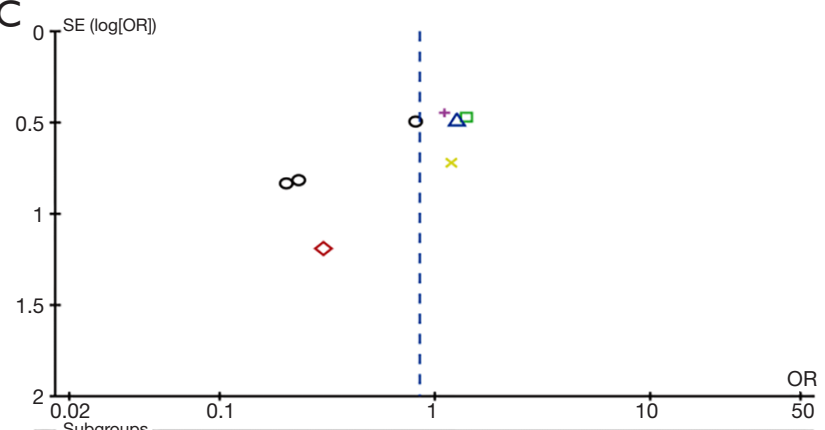

O Subgroups

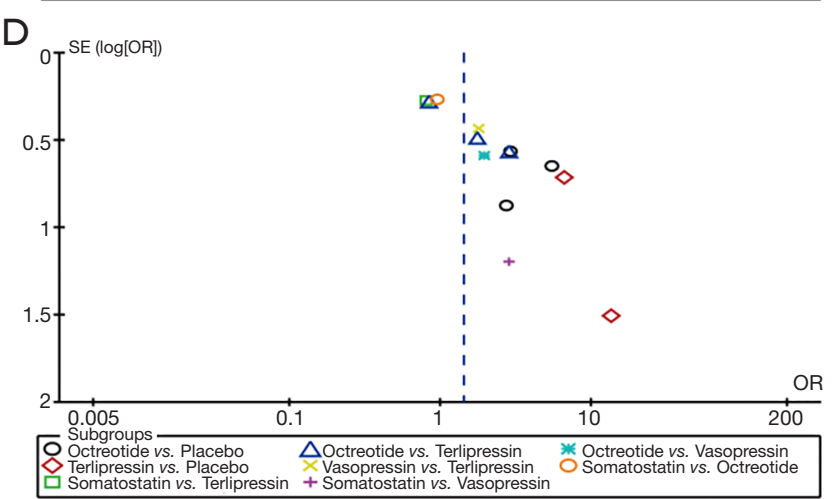

Figure S2 Funnel plots of 6-week mortality (A), five-day mortality (B), rebleeding (C) and control of initial bleeding (D). 
Table S1 Details of various search terms

Vasoconstrictor Agents AND (variceal band ligation OR endoscopic variceal ligation OR Sclerotherapy) AND Randomized Controlled Trial Somatostatin AND (variceal band ligation OR endoscopic variceal ligation OR Sclerotherapy) AND Randomized Controlled Trial Octreotide AND (variceal band ligation OR endoscopic variceal ligation OR Sclerotherapy) AND Randomized Controlled Trial Vasopressin AND (variceal band ligation OR endoscopic variceal ligation OR Sclerotherapy) AND Randomized Controlled Trial Terlipressin AND (variceal band ligation OR endoscopic variceal ligation OR Sclerotherapy) AND Randomized Controlled Trial Vasoactive drug AND (variceal band ligation OR endoscopic variceal ligation OR Sclerotherapy) AND Randomized Controlled Trial (Somatostatin OR Octreotide OR Vasopressin OR Terlipressin) AND endoscopy AND Randomized Controlled Trial 